\title{
Probing the photophysics of semiconductor nanomaterials using optical pump-terahertz probe spectroscopy: from nanowires to perovskites
}

Hannah J. Joyce, Lissa Eyre, Stephanie O. Adeyemo, Sarwat A. Baig, Jessica L. Boland, et al.

Hannah J. Joyce, Lissa Eyre, Stephanie O. Adeyemo, Sarwat A. Baig, Jessica L. Boland, Christopher L. Davies, Michael B. Johnston, Felix Deschler, H. Hoe Tan, C. Jagadish, "Probing the photophysics of semiconductor nanomaterials using optical pump-terahertz probe spectroscopy: from nanowires to perovskites," Proc. SPIE 10724, Physical Chemistry of Semiconductor Materials and Interfaces XVII, 107240F (7 September 2018); doi: 10.1117/12.2320720

EPIE Event: SPIE Nanoscience + Engineering, 2018, San Diego, California, United States 


\title{
Probing the photophysics of semiconductor nanomaterials using optical pump-terahertz probe spectroscopy: from nanowires to perovskites
}

\author{
Hannah J. Joyce* $^{* \mathrm{a}}$, Lissa Eyre ${ }^{\mathrm{a}}$, Stephanie O. Adeyemo ${ }^{\mathrm{a}}$, Sarwat A. Baig ${ }^{\mathrm{a}}$, Jessica L. Boland ${ }^{\mathrm{b}}$, \\ Christopher L. Davies $^{\mathrm{b}}$, Michael B. Johnston ${ }^{\mathrm{b}}$, Felix Deschler ${ }^{\mathrm{c}}$, H. Hoe Tan ${ }^{\mathrm{d}}$, C. Jagadish ${ }^{\mathrm{d}}$ \\ ${ }^{a}$ Department of Engineering, University of Cambridge, $9 \mathrm{JJ}$ Thomson Avenue \\ Cambridge CB3 0FA, United Kingdom; \\ ${ }^{\mathrm{b}}$ Department of Physics, University of Oxford, Clarendon Laboratory, Parks Road, \\ Oxford OX1 3PU, United Kingdom; \\ ${ }^{c}$ Department of Physics, University of Cambridge, Cavendish Laboratory, JJ Thomson Avenue, \\ Cambridge CB3 0HE, United Kingdom; \\ ${ }^{\mathrm{d} D e p a r t m e n t ~ o f ~ E l e c t r o n i c ~ M a t e r i a l s ~ E n g i n e e r i n g, ~ R e s e a r c h ~ S c h o o l ~ o f ~ P h y s i c s ~ a n d ~ E n g i n e e r i n g, ~}$ \\ The Australian National University, Canberra ACT 2601, Australia
}

\begin{abstract}
Optical pump-terahertz probe spectroscopy is a powerful contact-free technique for probing the electronic properties of novel nanomaterials and their response to photoexcitation. This technique can measure charge carrier transport and dynamics with sub-picosecond temporal resolution. Electrical conductivity, charge carrier lifetimes, mobilities, dopant concentrations and surface recombination velocities can be measured with high accuracy and with considerably higher throughput than achievable with traditional contact-based techniques. We describe how terahertz spectroscopy is revealing the fascinating properties and guiding the development of a number of promising semiconductor materials, with particular emphasis on III-V semiconductor nanowires and devices.
\end{abstract}

Keywords: Nanowire, III-V, semiconductor, terahertz, spectroscopy, ultrafast

\section{INTRODUCTION}

It is crucial, when developing optoelectronic devices based on novel nanomaterials, to have detailed knowledge of the electrical properties of the constituent nanomaterials. This knowledge, however, is often difficult to garner with conventional contact-based electrical measurements, owing to the small size and unconventional geometry of the nanomaterials. For example, III-V semiconductor nanowires are not amenable to Hall bar measurements due to their quasi-one-dimensional geometry. Optical pump-terahertz probe (OPTP) spectroscopy has emerged as a noncontact technique that is particularly well-suited for measuring a comprehensive set of electronic properties, including carrier lifetime, surface recombination velocity, ionised dopant concentration and carrier mobility in semiconductor nanomaterials. OPTP overcomes the difficulties associated with conventional contact-based electrical transport measurements, and it provides accurate measurements of charge carrier transport and dynamics at room temperature with subpicosecond temporal resolution. These terahertz $(\mathrm{THz})$ measurements are now guiding the development of a wide range of novel devices, such as nanowire-based and hybrid metal halide perovskite-based solar cells, ${ }^{1}$ nanowire transistors, ${ }^{2}$ ultrafast optically switchable polarisation modulators ${ }^{3}$ and photodetectors. ${ }^{4}$

*hannah.joyce@eng.cam.ac.uk; phone+44 1223748379

Physical Chemistry of Semiconductor Materials and Interfaces XVII, edited by Hugo A. Bronstein, Felix Deschler Proc. of SPIE Vol. 10724, 107240F · (C) 2018 SPIE · CCC code: 0277-786X/18/\$18 · doi: 10.1117/12.2320720 


\section{EXPERIMENTAL METHODS}

\subsection{Nanowire growth}

Prior to growth, (111)B substrates were treated with poly-L-lysine to enhance the adhesion of colloidal Au nanoparticles. Then a colloid of monodisperse Au nanoparticles of a particular size $(20,30,50 \mathrm{or} 80 \mathrm{~nm})$ was drop-cast on the substrate and the excess washed away with deionised water. The $\mathrm{Au}$ nanoparticles function to drive nanowire growth via a vapour-liquid-solid or vapour-solid-solid growth mechanism, and wet the growing tip of the nanowire. ${ }^{5}$ The nanoparticles are visible at the tips of the nanowires in the scanning electron microscope (SEM) images of Figure 1. Nanowires were grown by metalorganic chemical vapour deposition (MOCVD) in an Aixtron 200/4 horizontal flow reactor. The reactor pressure was 100 mbar and the total gas flow rate was $15 \mathrm{slm}$. The metalorganic precursors for the group III elements gallium, aluminium and indium were trimethylgallium, trimethylaluminium and trimethylindium, respectively. Arsine and phosphine were used as precursors for the group $\mathrm{V}$ elements arsenic and phosphorous, respectively.

Growth temperatures and the gas flow rates of the group III and V precursors were tuned to control the shape, crystal structure and electronic properties of the nanowires. Zinc-blende GaAs nanowires were grown using a two-temperature procedure involving a short high temperature $\left(450^{\circ} \mathrm{C}\right)$ nucleation step for $60 \mathrm{~s}$, followed by a longer growth step at a lower temperature $\left(375^{\circ} \mathrm{C}\right.$ to $\left.425^{\circ} \mathrm{C}\right)$. The high nucleation temperature enhances the yield of straight [111]B-oriented nanowires and the lower temperature reduces parasitic radial growth and supports the crystallisation of twin-free zincblende nanowires. ${ }^{6}$ A high V/III ratio was employed to further enhance the crystallization of the zinc-blende structure. ${ }^{7}$ Wurtzite GaAs nanowires were grown at a high temperature of $550{ }^{\circ} \mathrm{C}$ and a low V/III ratio of just 1.4. ${ }^{8}$ For GaAs/AlGaAs core-shell nanowires, zinc-blende GaAs cores were grown as described above and the AlGaAs shells were grown at a considerably higher temperature of 650 to $750{ }^{\circ} \mathrm{C}$, which favours radial shell growth. ${ }^{9}$ Zinc-blende InAs nanowires were grown at a low temperature $\left(400{ }^{\circ} \mathrm{C}\right)$ and a high V/III ratio. ${ }^{10}$ Wurtzite InAs nanowires (see Figure 1a) were achieved with a higher growth temperature $\left(500{ }^{\circ} \mathrm{C}\right.$ ) and low V/III ratio. ${ }^{10} \mathrm{InP}$ nanowires (see Figure $1 \mathrm{~b}$ ) were grown at $420^{\circ} \mathrm{C}$ and featured a predominately wurtzite crystal structure with a high density of zinc-blende insertions and stacking faults. ${ }^{11}$

After growth, vertical nanowires were transferred from the as-grown substrates to z-cut quartz for OPTP measurements. Quartz was chosen as the substrate because it has high transmittance between 0.1 and $3.7 \mathrm{THz}$ and it does not exhibit any response to photoexcitation.

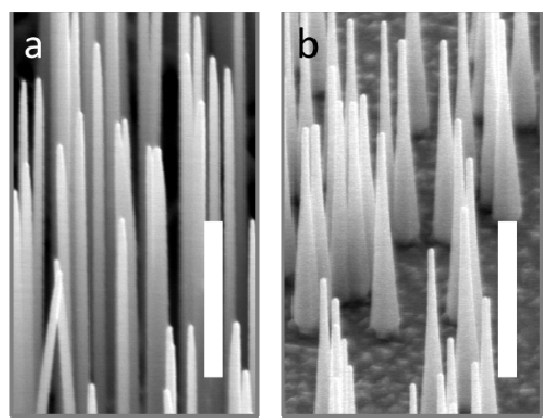

Figure 1. SEM images of (a) wurtzite InAs nanowires and (b) InP nanowires grown by MOCVD using Au nanoparticles to drive nanowire growth.

\subsection{Organometal trihalide perovskite film fabrication}

Solution-processed perovskite films were accomplished by spin-coating onto z-cut quartz substrates in a nitrogen-filled glovebox. The films were then annealed on a hotplate at temperatures between 70 and $150{ }^{\circ} \mathrm{C}$.

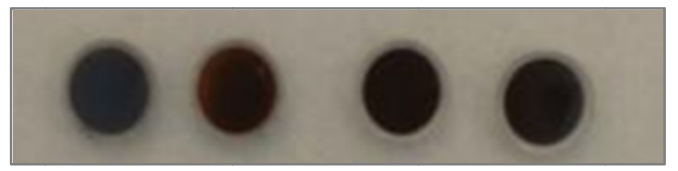

Figure 2. Photographs of methylammonium lead triiodide $\left(\mathrm{MAPbI}_{3}\right)$ films deposited on quartz for OPTP measurements. 


\subsection{Optical pump-terahertz probe spectroscopy}

All measurements were performed at room temperature and with the sample held in a vacuum box. An amplified Ti:Sapphire laser was used for $\mathrm{THz}$ generation, photoexcitation of the sample, and terahertz detection. The laser generated $35 \mathrm{fs}$ pulses centred at $800 \mathrm{~nm}$ at a $5 \mathrm{kHz}$ repetition rate and a pulse energy of $800 \mu \mathrm{J} / \mathrm{pulse}$. The laser beam was split into three paths so that $200 \mu \mathrm{J} /$ pulse was used to generate the $\mathrm{THz}$ probe pulse via optical rectification in a $2 \mathrm{~mm} \mathrm{GaP}$ crystal, $1.6 \mu \mathrm{J} /$ pulse was used as a gate for electro-optic detection of the transmitted $\mathrm{THz}$ pulse using a 200 $\mu \mathrm{m} \mathrm{GaP}$ crystal, and the remainder was used as the optical pump to photoexcite the sample at fluences between 1 and $280 \mu \mathrm{J} / \mathrm{cm}^{2}$. In most cases the $800 \mathrm{~nm}$ pulses were used to photoexcite the samples directly, but for wider bandgap samples (particularly perovskites), an optical parametric amplifier was used to generate higher energy photons. The $\mathrm{THz}$ probe measured an area of approximately constant photoexcited carrier density. The THz electric field, $E$, was detected using a balanced photodiode circuit connected to a lock-in amplifier, which was referenced to a chopper $(2.5 \mathrm{kHz})$ in the $\mathrm{THz}$ generation beam. To detect the photoinduced change in THz electric field, $\Delta E$, we used a second lock-in amplifier referenced to a second chopper $(125 \mathrm{~Hz})$ in the optical pump beam. The photoinduced change in the transmitted $\mathrm{THz}$ field, $\Delta E$, is directly proportional to the photoconductivity of the sample. The pulses used for THz generation and optical photoexcitation travelled via variable delay stages to vary the relative arrival times of the pulses. To obtain photoconductivity spectra at a given time after photoexcitation, we varied the delay between the terahertz probe and gate pulses. To obtain photoconductivity decays as a function of time after photoexcitation, we varied the delay between the optical pump and terahertz probe pulses.

\section{RESULTS AND DISCUSSION}

\subsection{Charge carrier lifetimes and surface recombination velocities}

Photoconductivity decays obtained from InAs and InP nanowires were well-fitted with single exponential functions, suggesting that non-radiative Shockley-Read-Hall recombination is the dominant recombination mechanism. The lifetimes of the InAs nanowires exhibited a strong dependence upon diameter, with narrow diameter InAs nanowires exhibiting shorter carrier lifetimes than nanowires with wider diameters. Table 1 lists the photoconductivity lifetimes for InAs nanowires of different diameters.

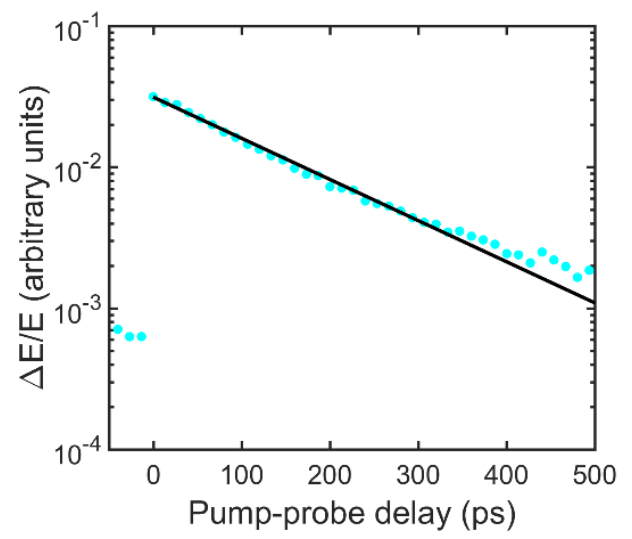

Figure 3. Photoconductivity decay of wurtzite InAs nanowires of $27 \mathrm{~nm}$ diameter. The cyan circles are the measured data points and the black line is a single exponential fit to the data. 
Table 1. Effect of nanowire diameter of photoconductivity lifetime for wurtzite InAs nanowires.

\begin{tabular}{|l|l|}
\hline \multicolumn{1}{|c|}{$\begin{array}{c}\text { Diameter } \\
(\mathbf{n m})\end{array}$} & $\begin{array}{c}\text { Photoconductivity lifetime } \\
\text { (ps) }\end{array}$ \\
\hline 27 & 200 \\
\hline 45 & 290 \\
\hline 95 & 470 \\
\hline 195 & 660 \\
\hline
\end{tabular}

This diameter-dependence arises due to the dominance of surface recombination in nanowires with narrow diameters. By approximating the nanowire as an infinite cylinder and solving the continuity equation for diffusion and recombination, we obtain the following relationship between photoconductivity lifetime $\tau$ and nanowire diameter, $d$ :

$$
\frac{1}{\tau}=\frac{1}{\tau_{v}}+\frac{4 S}{d}
$$

Here $\tau_{\mathrm{v}}$ is the lifetime in bulk material and $S$ is the surface recombination velocity. By fitting Equation 1 to the data in Table 1, we obtain a value for the surface recombination velocity in wurtzite InAs nanowires of $S=3.0 \times 10^{3} \mathrm{~cm} / \mathrm{s}^{12} \mathrm{In}$ contrast, the photoconductivity decays of InP nanowires did not exhibit strong dependence on diameter. This indicates a very low surface recombination velocity in the InP nanowires, which was calculated as $S=170 \mathrm{~cm} / \mathrm{s}^{13}$

The photoconductivity decays of GaAs nanowires exhibit a strong dependence on nanowire diameter, but unlike InAs and InP nanowires were not single exponential. ${ }^{14}$ Furthermore, in GaAs nanowires the decay rate slows with increasing photoexcitation fluence and with increasing time after photoexcitation. ${ }^{15}$ These two observations indicate that the surface traps are saturable. We fitted the data with a pair of coupled rate equations describing the carrier concentration, $N$, and the surface density of unfilled traps, $T$. The maximum surface recombination velocity, which occurs in the limit of all traps being empty, was calculated to be $1.3 \times 10^{6} \mathrm{~cm} / \mathrm{s}$ and the minimum surface recombination velocity in the limit of all traps being full was calculated to be $1.7 \times 10^{5} \mathrm{~cm} / \mathrm{s}^{14}$

We then optimised the growth of AlGaAs shells to achieve surface passivation of GaAs nanowires and thereby enhance charge carrier lifetimes in GaAs/AlGaAs core-shell nanowires. ${ }^{15,16}$ Drastic improvements in photoconductivity lifetime were observed with increasing Al composition and increasing the thickness of the shell, as indicated in Table $2 .{ }^{17}$

Table 2. Effect of $\mathrm{Al}_{x} \mathrm{Ga}_{1-x} \mathrm{As}$ shell composition and thickness on the photoconductivity lifetime of GaAs/AlGaAs coreshell nanowires.

\begin{tabular}{|c|c|c|}
\hline $\begin{array}{c}\text { Al composition, } \boldsymbol{x} \\
(\mathbf{\%})\end{array}$ & $\begin{array}{c}\text { Shell thickness } \\
(\mathbf{n m})\end{array}$ & $\begin{array}{c}\text { Photoconductivity } \\
\text { lifetime } \\
\text { (ps) }\end{array}$ \\
\hline 26 & 30 & 8 \\
\hline 50 & 5 & 650 \\
\hline 50 & 10 & 900 \\
\hline 50 & 16 & 1100 \\
\hline 50 & 34 & 1300 \\
\hline
\end{tabular}




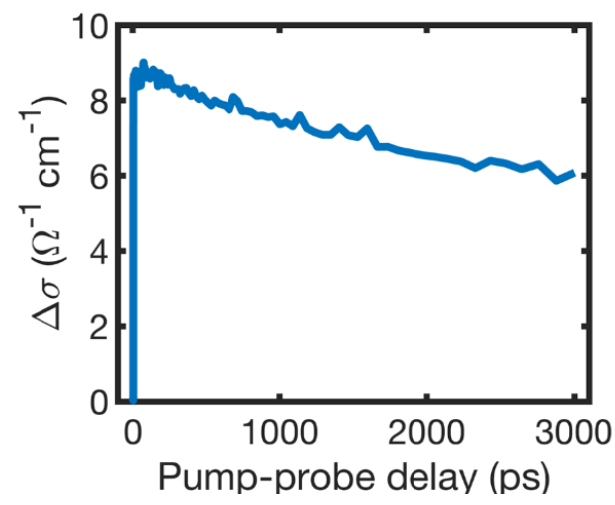

Figure 4. Photoconductivity decay measured for organometal trihalide perovskite film after photoexcitation with a pulse of wavelength $740 \mathrm{~nm}(1.7 \mathrm{eV})$ and fluence of $80 \mu \mathrm{J} / \mathrm{cm}^{2}$.

Photoconductivity dynamics in organometal trihalide perovskites (e.g. Figure 4) show dependence upon photoexcited carrier density and time after photoexcitation. These photoconductivity decays are well modelled by rate equations that include Auger recombination, band-to-band recombination and Shockley-Read-Hall recombination. ${ }^{18}$

\subsection{Photoconductivity spectra}

Photoconductivity spectra of all nanowire samples exhibited a pronounced Lorentzian response, as is characteristic of plasmon modes in weakly and moderately-doped semiconductors nanostructures. ${ }^{12}$ To these spectra we fitted Lorentzian functions with the electron scattering rate, $\gamma$, as a fitting parameter. ${ }^{12,13}$ The electron scattering rate can be converted to electron mobility via

$$
\mu=\frac{q}{m_{e}^{*} \gamma},
$$

where $m_{e}{ }^{*}$ is the electron effective mass and $q$ is the electronic charge. Unpassivated GaAs nanowires featured diameterdependent electron mobilities between 400 and $2100 \mathrm{~cm}^{2} \mathrm{~V}^{-1} \mathrm{~s}^{-1} .{ }^{14}$ The electron mobility decreased substantially with decreasing nanowire diameter as charge carriers experience increased scattering at nanowire surfaces. Passivation with AlGaAs shells improved the mobility to within $65 \%$ of the value expected for high quality bulk GaAs. ${ }^{17}$

\section{CONCLUSIONS}

OPTP provides accurate measurements of charge carrier transport and dynamics at room temperature with subpicosecond temporal resolution. It does not require electrical contacts, and therefore is ideal for samples such as nanowires that are difficult to measure by conventional contact-based methods. It also does not require the samples to be luminescent, and is therefore suitable for samples with low quantum efficiency and short charge carrier lifetimes.

\section{ACKNOWLEDGEMENTS}

The authors gratefully acknowledge financial support from the European Research Council (ERC Starting Grant ACrossWire), the Engineering and Physical Sciences Research Council (UK), the Australian Research Council, and the Australian National Fabrication Facility (ANFF). H. J. Joyce thanks the Royal Commission for the Exhibition of 1851 for her research fellowship. 


\section{REFERENCES}

[1] Zhong, Z., Li, Z., Gao, Q., Li, Z., Peng, K., Li, L., Mokkapati, S., Vora, K., Wu, J., Zhang, G., Wang, Z., Fu, L., Tan, H. H. and Jagadish, C., "Efficiency enhancement of axial junction InP single nanowire solar cells by dielectric coating," Nano Energy 28, 106-114 (2016).

[2] Alexander-Webber, J. A., Groschner, C. K., Sagade, A. A., Tainter, G., Gonzalez-Zalba, M. F., Pietro, R. Di, Wong-leung, J., Tan, H. H., Jagadish, C., Hofmann, S. and Joyce, H. J., "Engineering the Photoresponse of InAs Nanowires," ACS Appl. Mater. Interfaces 9, 43993-44000 (2017).

[3] Baig, S. A., Boland, J. L., Damry, D. A., Tan, H. H., Jagadish, C., Joyce, H. J. and Johnston, M. B., “An Ultrafast Switchable Terahertz Polarization Modulator Based on,” Nano Lett. 17, 2603-2610 (2017).

[4] Peng, K., Parkinson, P., Fu, L., Gao, Q., Jiang, N., Guo, Y.-N., Wang, F., Joyce, H. J., Boland, J. L., Tan, H. H., Jagadish, C. and Johnston, M. B., "Single nanowire photoconductive terahertz detectors," Nano Lett. 15(1), 206210 (2015).

[5] Jiang, N., Wong-Leung, J., Joyce, H. J., Gao, Q., Tan, H. H. and Jagadish, C., "Understanding the true shape of Au-catalyzed GaAs nanowires," Nano Lett. 14(10), 5865-5872 (2014).

[6] Joyce, H. J., Gao, Q., Tan, H. H., Jagadish, C., Kim, Y., Zhang, X., Guo, Y. and Zou, J., “Twin-free uniform epitaxial GaAs nanowires grown by a two-temperature process," Nano Lett. 7(4), 921-926 (2007).

[7] Joyce, H. J., Gao, Q., Tan, H. H., Jagadish, C., Kim, Y., Fickenscher, M. A., Perera, S., Hoang, T. B., Smith, L. M., Jackson, H. E., Yarrison-Rice, J. M., Zhang, X. and Zou, J., "High purity GaAs nanowires free of planar defects: Growth and characterization," Adv. Funct. Mater. 18(23), 3794-3800 (2008).

[8] Joyce, H. J., Gao, Q., Wong-Leung, J., Kim, Y., Tan, H. H. and Jagadish, C., “Tailoring GaAs, InAs, and InGaAs nanowires for optoelectronic device applications," IEEE J. Sel. Top. Quantum Electron. 17(4), 766-778 (2011).

[9] Jiang, N., Parkinson, P., Gao, Q., Breuer, S., Tan, H. H., Wong-Leung, J. and Jagadish, C., "Long minority carrier lifetime in Au-catalyzed GaAs/AlxGa1-xAs core-shell nanowires," Appl. Phys. Lett. 101(2), 23111 (2012).

[10] Joyce, H. J., Wong-Leung, J., Gao, Q., Hoe Tan, H. and Jagadish, C., "Phase perfection in zinc blende and wurtzite III-V nanowires using basic growth parameters," Nano Lett. 10(3), 908-915 (2010).

[11] Paiman, S., Gao, Q., Joyce, H. J., Kim, Y., Tan, H. H., Jagadish, C., Zhang, X., Guo, Y. and Zou, J., “Growth temperature and V/III ratio effects on the morphology and crystal structure of InP nanowires," J. Phys. D. Appl. Phys. 43(44), 445402 (2010).

[12] Joyce, H. J., Docherty, C. J., Gao, Q., Tan, H. H., Jagadish, C., Lloyd-Hughes, J., Herz, L. M. and Johnston, M. B., "Electronic properties of GaAs, InAs and InP nanowires studied by terahertz spectroscopy.," Nanotechnology 24(21), 214006 (2013).

[13] Joyce, H. J., Wong-Leung, J., Yong, C.-K., Docherty, C. J., Paiman, S., Gao, Q., Tan, H. H., Jagadish, C., LloydHughes, J., Herz, L. M. and Johnston, M. B., "Ultralow surface recombination velocity in InP nanowires probed by terahertz spectroscopy," Nano Lett. 12(10), 5325-5330 (2012).

[14] Joyce, H. J., Baig, S. A., Parkinson, P., Davies, C. L., Boland, J. L., Tan, H. H., Jagadish, C., Herz, L. M. and Johnston, M. B., "The influence of surfaces on the transient terahertz conductivity and electron mobility of GaAs nanowires," J. Phys. D. Appl. Phys. 50(22) (2017).

[15] Parkinson, P., Joyce, H. J., Gao, Q., Tan, H. H., Zhang, X., Zou, J., Jagadish, C., Herz, L. M. and Johnston, M. B., "Carrier lifetime and mobility enhancement in nearly defect-free core-shell nanowires measured using timeresolved terahertz spectroscopy," Nano Lett. 9(9), 3349-3353 (2009).

[16] Titova, L. V., Hoang, T. B., Jackson, H. E., Smith, L. M., Yarrison-Rice, J. M., Kim, Y., Joyce, H. J., Tan, H. H. and Jagadish, C., "Temperature dependence of photoluminescence from single core-shell GaAs-AlGaAs nanowires," Appl. Phys. Lett. 89(17), 173126 (2006). 
[17] Joyce, H. J., Parkinson, P., Jiang, N., Docherty, C. J., Gao, Q., Tan, H. H., Jagadish, C., Herz, L. M. and Johnston, M. B., "Electron mobilities approaching bulk limits in 'surface-free' GaAs nanowires," Nano Lett. 14(10), 5989-5994 (2014).

[18] Wehrenfennig, C., Eperon, G. E., Johnston, M. B., Snaith, H. J. and Herz, L. M., "High charge carrier mobilities and lifetimes in organolead trihalide perovskites," Adv. Mater. 26(10), 1584-1589 (2014). 